Hautarzt 2011 · 62:265-265

DOI 10.1007/s00105-010-2072-y

Online publiziert: 25. März 2011

(c) Springer-Verlag 2011

\author{
M. Meurer \\ Universitäts AllergieCentrum (UAC), Universitätsklinikum Carl \\ Gustav Carus an der Technischen Universität Dresden
}

\title{
Atypische Mykobakteriosen
}

Die Erkennung von Infektionen durch „atypische“, d. h. nichttuberkulöse Mykobakterien (NTM) ist aufgrund der ständig wachsenden Anzahl von betroffenen, immunkompetenten wie immunsupprimierten Patienten von aktueller medizinischer Bedeutung.

Während disseminierte oder lokalisierte extrapulmonale Verlaufsformen überwiegend bei stark immungeschwächten Patienten auftreten und in 90\% durch den Mycobacterium-avium-Komplex bedingt sind - wie der Beitrag von Dr. Herzmann et al. aus Borstel und Essen hervorhebt -, werden die rein kutanen Verlaufsformen in unseren Breiten v. a. durch schnell wachsende NMT wie $M$. fortuitum oder durch M. marinum, dem Erreger des Schwimmbadgranuloms, hervorgerufen. Der Leitthemenbeitrag von Prof. Nenoff et al. aus Leipzig konzentriert sich auf diesen für uns Dermatologen besonders wichtigen Erreger.

Das klinische Bild der kutanen NTMInfektionen ist außerordentlich vielgestaltig und häufig ebenfalls „atypisch“. Eine gezielt erfragte Anamnese mit Angaben über wiederholte Kontakte zu kontaminiertem Wasser bzw. über vorausgegangene Bagatelltraumen, Injektionen, Impfungen oder unsteril durchgeführte Akupunktur oder Mesotherapie führt häufig auf die richtige diagnostische Spur.

Beide Leitthemenbeiträge betonen die Wichtigkeit einer lege artis durchgeführten mikrobiologischen Diagnostik zur Sicherung der Diagnose einer Hautinfektion mit NTM.

Die antibiotische Mono- oder ggf. Kombinationstherapie der NTM-Infektion erfordert - v. a. bei immunsupprimierten Patienten, wie der Beitrag von
Dr. Herzmann et al. zeigt - Erfahrung und häufig Geduld. Die Konzentration auf 2 Beiträge ermöglicht den Leserinnen und Lesern dieses Leitthemenheftes von Der Hautarzt eine rasche und übersichtliche Information über die aktuellen $\mathrm{Be}$ handlungsmöglichkeiten kutaner NTMInfektionen.

Ich bin beiden Autorenteams für die kollegiale Bereitstellung und die hervorragende Qualität ihrer Beiträge, die zweifellos großes Interesse finden werden, außerordentlich dankbar.

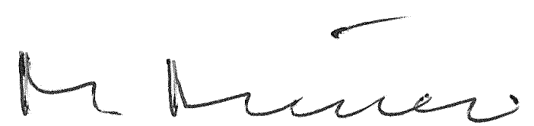

Prof. Michael Meurer

\section{Korrespondenzadresse \\ Prof. Dr. M. Meurer}

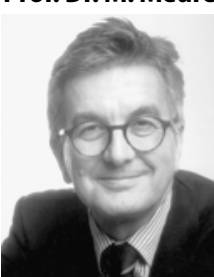

Universitäts AllergieCentrum (UAC), Universitätsklinikum Carl Gustav Carus an der Technischen Universität Dresden Fetscherstr. 74, 01307 Dresden michael.meurer@uniklinikum-dresden.de 